\title{
BMC Pulmonary Medicine reviewer acknowledgement 2014
}

\section{Catia Cornacchia}

\section{Carrie Aaron}

USA

John Abisheganaden

Singapore

Simone Accordini

Italy

Ritesh Agarwal

India

Daniel Ajona

Spain

Mohammed Al Ghobain

Saudi Arabia

Mazen Al-Alawi

Ireland

Pedro Almagro

Spain

Jan Peder Amlie

Norway

Benoit Arnould

France

Michael Arzt

Germany

Elie Azoulay

France

Helena Backman

Sweden

Mona Bafadhel

UK

Ulas Bagci

USA

\author{
Stefano Baglioni \\ Italy
}

Chunxue Bai

China

Emma Baker

UK

Ioannis Bakolis

UK

Stefan Balabanov

Switzerland

Rui Baptista

Portugal

Olga Barbarsh

Russian Federation

Gabriele Bassi

Italy

Eric Bateman

South Africa

Jeremy Beitler

USA

Kathleen Bennett

Ireland

Pascal Berna

France

Laurent Bertoletti

France

Rajesh Bhagat

USA

Urvashi Bhan

USA
Surya Bhatt

USA

Andrea Bianco

Italy

Lucie Blais

Canada

Konrad Bloch

Switzerland

Jessica Bon

USA

Francesco Bonella

Germany

Martina Bonifazi

Italy

Berislav Bosnjak

Austria

Ynuk Bosse

Canada

Apostolos Bossios

Sweden

Stephen Bourke

UK

\section{Afroditi Boutou}

Greece

Fulvio Braido

Italy

Evan Brittain

USA

Raquel Britto

Brazil

Correspondence: Catia.Cornacchia@biomedcentral.com

BioMed Central, Floor 6, 236 Gray's Inn Road, London WC1X 8HB, UK 


\begin{tabular}{|c|c|c|}
\hline Malcolm Brodlie & Helen Chapel & Roberto Dantas \\
\hline UK & UK & Brazil \\
\hline Denise Brookes & Kenneth Chapman & Kaid Darwiche \\
\hline Australia & Canada & Germany \\
\hline Dina Brooks & Davide Chiumello & Theodore Dassios \\
\hline Canada & Italy & UK \\
\hline Antonio Bugalho & Chang Won Choi & Warren Davidson \\
\hline Portugal & Korea, South & Canada \\
\hline Pierre-Regis Burgel & Sanjay Chotirmall & Wilfried De Backer \\
\hline France & Ireland & Belgium \\
\hline Jennifer Butcher & Shah-Hwa Chou & Francesco De Blasio \\
\hline USA & Taiwan & Italy \\
\hline Scotty Butcher & Antonio Clavenna & Anthony De Soyza \\
\hline Canada & Italy & UK \\
\hline Elif Cadirci & Amelia Clive & Esther De Vries \\
\hline Turkey & UK & Netherlands \\
\hline Carlo Caffarelli & Paola Cogo & Chris Del Mar \\
\hline Italy & Italy & Australia \\
\hline Shaoxi Cai & Harold Collard & Christophe Delclaux \\
\hline China & USA & France \\
\hline Luigino Calzetta & Adam Collison & Yoshiki Demura \\
\hline Italy & Australia & Japan \\
\hline Bin Cao & Richard Conway & Olufemi Desalu \\
\hline China & Ireland & Nigeria \\
\hline Vera Luiza Capelozzi & Liam Cormican & Gaetan Deslee \\
\hline Brazil & Ireland & France \\
\hline Ralph Caraballo & Antonio Corrado & Harshad Devarbhavi \\
\hline USA & Italy & India \\
\hline Michele Caraglia & Cristina Costa & Philippe Devillier \\
\hline Italy & Italy & France \\
\hline Gaetano Caramori & Eduardo Costa & Philip Diaz \\
\hline Italy & Brazil & USA \\
\hline Annalisa Carlucci & David Coultas & Simon Doe \\
\hline Italy & USA & UK \\
\hline Maria Carrabba & Bianca Cox & Arnau Domenech \\
\hline Italy & Belgium & Netherlands \\
\hline Lucia Cazzoletti & Narelle Cox & Frank Domino \\
\hline Italy & Australia & USA \\
\hline Adrian Ceccato & Bruno Crestani & Massimiliano Don \\
\hline Argentina & France & Italy \\
\hline James Chalmers & Jane Cross & James Donohue \\
\hline UK & UK & USA \\
\hline Mathias Chamaillard & Elliot Crouser & Simone Dore \\
\hline France & USA & Italy \\
\hline
\end{tabular}


Victor Dourado

Brazil

Silvano Dragonieri

Italy

Mark Dransfield

USA

Gordon Drummond

UK

Orianne Dumas

France

\section{Steven Duncan}

USA

Anthony D'Urzo

Canada

Michelle Eakin

USA

Anna Eis-Huebinger

Germany

Mohamed Elloumi

Tunisia

John Engelhardt

UK

Ralph Epaud

France

Berne Eriksson

Sweden

Joan Escarrabill

Spain

Francisco Estévez

Spain

Mark Everard

Australia

Shirley Fabris De Souza

Brazil

Alen Faiz

Netherlands

Francesco Fanfulla

Italy

Fatemeh Fattahi

Netherlands

Brigitte Fauroux

France

Cristiano Fava

Italy
Ademola Fawibe

Nigeria

Niki Fens

Netherlands

De Benedetto Fernando

Italy

Miguel Ferrer

Spain

James Fink

USA

Claudia Flexeder

Germany

Gert Folkerts

Netherlands

Marilyn Foreman

USA

Stephen Fowler

UK

Angela Frank

USA

Karl-Josef Franke

Germany

Oren Fruchter

Israel

En-Qing Fu

China

Kazuki Furuhashi

Singapore

Taiji Furukawa

Japan

Jacques Gaillat

France

Miguel Gallego

Spain

Gilles Garcia

France

Vanessa Garcia Larsen

UK

Gail Gauvreau

Canada

Chris Gentry

USA

Johnson George

Australia
William Gerthoffer

USA

Giovanni Gherardi

Italy

Lisa Giovannini-Chami

France

John Gjevre

Canada

Rainer Gloeckl

Germany

Gabriela Godaly

Sweden

Irma Godoy

Brazil

Tomasz Golczewski

Poland

Lutz Goldbeck

Germany

Monica Goldklang

USA

Matteo Goldoni

Italy

Donna Goodridge

Canada

Brian Graham

Canada

Ronda Greaves

Australia

Catherine Greene

Ireland

Matthias Griese

Germany

Wolfgang Gruber

Germany

Jordan Guenette

Canada

Ashleigh Guillaumier Australia

Ersin Gunay

Turkey

Mingzhou Guo

China

Henrik Gutte

Denmark 
Stig Hagstad

Sweden

Huang Haibo

China

Peter Hallas

Denmark

Sophie Hambleton

UK

Megan Hardin

USA

Kim Hare

Australia

Dominik Hartl

Germany

Apostolia Hatziefthimiou

Greece

\section{Andreas Hector}

Germany

Linnea Hedman

Sweden

Craig Hersh

USA

Genevieve Hery-Arnaud

France

Karen Heslop

Catherine Hill

Australia

Karen Hind

UK

Jonathan Hobson

UK

Susan Hodgson

UK

Anne Holland

Australia

Sakae Homma

Japan

Arie Hoogendijk

Netherlands

Nobuyuki Horita

Japan

Wen-Ni Huang

Taiwan
Kewu Huang

China

Martin Hug

Germany

Rodney Hughes

UK

Erik Hulzebos

Netherlands

Naoki Inagaki

Japan

Naohiro Inohara

USA

José Luis Izquierdo Alonso

Spain

David Jacobs

USA

Alan James

Australia

An Soo Jang

Korea, South

Susan Janson

USA

Thomas Janssens

Belgium

Berthold Jany

Germany

Dennis Jensen

Canada

Cheng Ji

USA

Wang Jianchun

China

Ane Johannessen

Norway

Sebastian Johnston

UK

Paul Jones

UK

J. Joseph

USA

Riitta Kaarteenaho

Finland

Hannu Kankaanranta

Finland
Stefan Karrasch

Germany

Nikoleta Kartali

Greece

Parastu Kasaie

USA

Bernet Kato

UK

Leslie Katzel

USA

Thomas Keil

Germany

Vanessa Kelly

USA

Brian Kent

UK

H.A.M. Kerstjens

Netherlands

Edward Kerwin

USA

Rainer Kiefmann

Germany

Jae Yeol Kim

Korea, South

Victor Kim

USA

Paul King

Australia

Sylvia Knapp

Austria

Lars Knudsen

Germany

Andreas Rembert Koczulla

Germany

Manolis Kogevinas

Spain

Efstratios Koletsis

Greece

Jay Kolls

USA

Christos Kontogiorgis

Greece

Jukka Koskela

Finland 
Konstantinos Kostikas

Greece

Michael Kreuter

Germany

Jerry Krishnan

USA

Annemarije Kruis

Netherlands

Hiroshi Kubo

Japan

Tejaswini Kulkarni

USA

Rakesh Kumar

Australia

Anna Kurdowska

USA

Jessica Kynyk

USA

Larry Lands

Canada

Arnulf Langhammer

Norway

Pierantonio Laveneziana

France

Federico Lavorini

Italy

Lee Lay Tin

Singapore

Solène Le Gal

France

David Lederer

USA

T-C Lee

Taiwan

Augustine Lee

USA

Anke Lenferink

Netherlands

Steffen Leonhardt

Germany

Christophe Leroyer

France

Janice Leung

Canada
James Lewis

USA

Yongde Liao

China

Yick Hou Lim

Singapore

Hsing-Lin Lin

Taiwan

Ann Lindberg

Sweden

Kathleen Lindell

USA

Jianhong Liu

China

Zhangsuo Liu

China

Adrian Loerbroks

Germany

Anders Løkke

Denmark

Emmanuel Lorne

France

Teck Boon Low

Singapore

Renzo Loyaga-Rendon

USA

Thomas Luecke

Germany

Bo Lundbã $f \hat{A} a C k$

Sweden

Alex Mackay

UK

Kenneth Macleod

UK

Stephanie Macneill

UK

Brendan Madden

UK

Jesper Magnusson

Sweden

Rohan Maharaj

Trinidad and Tobago

Sara Maio

Italy
Subramaniam Malarkannan

USA

Fabien Maldonado

USA

Giovanni Malerba

Italy

Manoj Mammen

USA

Lyndon Mansfield

UK

Emilio Marangio

Italy

Nathaniel Marchetti

USA

Darcy Marciniuk

Canada

Alessandro Marcon

Italy

Tatiana Maron-Gutierrez

Brazil

Lorraine Martin

UK

Carlos Martinez

USA

Miguel Angel Martinez-Garcia

Spain

Shin Matsuoka

Japan

Joerg Mattes

Australia

John Matthews

USA

Oscar Mayer

USA

David Mcallister

UK

Amanda Mccullough

Australia

Sharon Mcgrath-Morrow

USA

Stephen Mckenna

UK

Darren Mcloughlin

Ireland 
Brent Mcparland

Australia

Sanjay Mehta

Canada

Silke Meiners

Germany

Spyros Mentzelopoulos

Greece

Elodie Merlot

France

Alessandro Mezzani

Italy

Massimo Miniati

Italy

Marc Miravitlles

Spain

Giuseppe Miserocchi

Italy

Howard Mitchell

Australia

Susanne Modrow

Germany

Kelly Moffitt

UK

Seyed Javad Moghaddam

USA

Firdaus Aa Mohamed Hoesein

Netherlands

Geraldine Moloney

Ireland

Ute Mons

Germany

Paolo Montuschi

Italy

Norman Morris

Australia

Stephen Mshana

Tanzania

Magdalena Muc

Poland

Hiroshi Mukae

Japan

David Murdoch

New Zealand
Nicola Murgia

Sweden

Vanessa Murphy

Australia

Michelle Murray

Ireland

Marjukka Myllarniemi

Finland

Ernest Nadal

USA

Lutz Naehrlich

Germany

Prasad Nagakumar

UK

Atsushi Nambu

Japan

Steven Nathan

USA

J Alberto Neder

Brazil

Nicholas Newman

USA

Heber Nielsen

USA

Vance Nielsen

USA

Carlos Nigro

Argentina

Akio Niimi

Japan

Peter B Noble

Australia

Hiroaki Nomori

Japan

Cathal O Broin

Ireland

Oisin O'Connell

Ireland

Denis O'Donnell

Canada

Shinichiro Ohshimo

Japan

Brian Oliver

Australia
Mario Olivieri

Italy

Shane O'Neill

Ireland

Ellie Oostveen

Belgium

David Orenstein

USA

Stylianos Orfanos

Greece

Adeola Orogade

Nigeria

Christian Osadnik

Australia

Caroline Owen

USA

Panagiotis Paliogiannis

Italy

G Iyer Parameswaran

USA

Hae-Sim Park

Korea, South

Edwin Roger Parra

Brazil

Christopher Pascoe

Canada

Franco Pasqua

Italy

Neeti Pathare

USA

Rodolfo Paula Vieira

Brazil

Claudio Pedone

Italy

Tatjana Pejcic

Serbia

Véronique Pepin

Canada

Eanes Pereira

Brazil

Antonio Pereira-Vega

Spain

Diego Peroni

Italy 


\begin{tabular}{|c|c|c|}
\hline Lionel Perrier & Monika Raulf-Heimsoth & Sejal Saglani \\
\hline France & Germany & UK \\
\hline Irina Petrache & Nicolas Regamey & Amali Samarasinghe \\
\hline USA & Switzerland & USA \\
\hline Claudio Pignata & Kyndaron Reinier & Takuya Samukawa \\
\hline Italy & USA & Japan \\
\hline Mariona Pinart & Marie-Pierre Revel & Claudio Maria Sanguinetti \\
\hline Spain & France & Italy \\
\hline Victor Pinto-Plata & Julian Rex & Pierachille Santus \\
\hline USA & Sweden & Italy \\
\hline Ruben Pio & Biarta Rhys-Jones & Ian Sayers \\
\hline Spain & Australia & UK \\
\hline Rein Jan Piso & Matija Rijavec & Paul Scanlon \\
\hline Switzerland & Slovenia & USA \\
\hline Tainá Pizzignacco & Ger Rijkers & Tjard Schermer \\
\hline Brazil & Netherlands & Netherlands \\
\hline Venerino Poletti & Jeremy Road & Tamara Schikowski \\
\hline Italy & Canada & Switzerland \\
\hline Janos Porszasz & Fatima Rodrigues & Kerry Schnell \\
\hline USA & Portugal & USA \\
\hline David Price & Josanna Rodriguez & Clara Schroedl \\
\hline UK & USA & USA \\
\hline Mariano Provencio & Juan Carlos Rodriguez & Erica Schultz \\
\hline Spain & Spain & Sweden \\
\hline Nirupama Putcha & Micaela Romagnoli & Richard Schulz \\
\hline USA & Italy & Germany \\
\hline Biyun Qian & Robert Ross & Susanne Schulz \\
\hline China & USA & Germany \\
\hline Xiaoqun Qin & Harry Rossiter & Hayley Scott \\
\hline China & USA & Australia \\
\hline Stuart Quan & Rhonda Rosychuk & Ikuo Sekine \\
\hline USA & USA & Japan \\
\hline Philip Quanjer & Kjetil Roth & Jacobo Sellares \\
\hline Netherlands & Norway & Spain \\
\hline Alexandra L. Quittner & Maureen Rutten-Van Molken & David Serisier \\
\hline USA & Netherlands & Australia \\
\hline Bradley Quon & Olli Ruuskanen & Waheed Shabbir \\
\hline Canada & Finland & Austria \\
\hline Danuta Radzioch & Dermot Ryan & Mohamed Shamji \\
\hline Canada & UK & UK \\
\hline S.Vamsee Raju & Christopher Ryerson & Nikhil Sharma \\
\hline USA & Canada & India \\
\hline Fanny Rancière & Didier Saey & Sk Sharma \\
\hline France & Canada & India \\
\hline
\end{tabular}


Dominick Shaw

UK

Han-Ming Shen

Singapore

Kartik Shenoy

USA

Guochao Shi

China

Minfeng Shu

USA

Chin-Chung Shu

Taiwan

Nikos Siafakas

Greece

Lori Silveira

USA

Donald Silverberg

Israel

Patricia Silveyra

USA

Jodie Simpson

Australia

Sally Singh

UK

Marcus Sjödin

Sweden

Szymon Skoczynski

Poland

Chris Smerecnik

Netherlands

Elizabeth Soares

Brazil

Xavier Soler

USA

Paolo Solidoro

Italy

Jinwoo Song

USA

Ulrich Specks

USA

Malcolm Starkey

Australia

Brian Stein

USA
Sigurd Steinshamn

Norway

Iwona Stelmach

Poland

Nikolai Stenfors

Sweden

Francois Stephan

France

Martina Sterclova

Czech Republic

Peter Sterk

Netherlands

Alastair Stewart

Australia

Kerstin Ström

Sweden

Hiromu Suzuki

Japan

Nicola Sverzellati

Italy

Steve Swain

USA

Tomasz Szul

USA

Toshinori Takada

Japan

Nagio Takigawa

Japan

Patricia Talamás-Rohana

Mexico

Hiroshige Taniguchi

Japan

Claudio Tantucci

Italy

Lisete Teixeira

Brazil

Paula Tejera

USA

Rachel Tham

Australia

Signe Timm

Denmark

Rabindra Tirouvanziam

USA
Nevins Todd

USA

Yuji Tohda

Japan

Sara Tomassetti

Italy

Corey Tomczak

Canada

Takahiro Tsuburai

Japan

Alice Turner

UK

Anne Therese Tveter

Norway

Jean Tyrrell

USA

Argyris Tzouvelekis

Greece

John Upham

Australia

Don Urquhart

UK

Samuel Valenca

Brazil

Françoise Van Bambeke

Belgium

Bram Van Den Borst

Netherlands

Arnoldus J.R. Van Gestel

Switzerland

Rebecca Vanders

Australia

Ruud Veldhuizen

Canada

Mario Venditti

Italy

Alain Vergnenègre

France

Diego Viasus

Colombia

Eugene Haydn Walters

Australia

Peter Wark

Australia 
George Washko

USA

Elizabeth Wasilevich

USA

Tomohiro Watanabe Japan

Steven Weinstein

USA

James Michael Wells

USA

Anja Werno

New Zealand

Geertjan Wesseling

Netherlands

Jiri Widimsky

Czech Republic

Ursula Winzer-Serhan

USA
Lisa Wood

Australia

Heinrich Worth

Germany

Tsuneo Yamashiro

Japan

Ian Yang

Australia

Ming Yang

Australia

Jun Yokota

Spain

Robert P Young

New Zealand

Susan Yount

USA

Zafar Zafari

Canada
Warren Zapol

USA

Paul Zarogoulidis

Greece

Qingxiang Zeng

Australia

Jie Zhang

China

Lin Zhang

USA

Graeme Zosky

Australia

Biljana Zvezdin

Serbia 